\title{
A Nomogram Model Based on Enhanced CT Radiomics Features for Predicting Tumor Response to Chemoradiotherapy in Patients With Esophageal Squamous Cell Carcinoma
}

\section{Jie Kong}

Hebei Medical University Fourth Affiliated Hospital and Hebei Provincial Tumor Hospital

\section{Liang Chen}

Shijiazhuang Obstetrics and Gynecology Hospital

\section{Shuchai Zhu}

Hebei Medical University Fourth Affiliated Hospital and Hebei Provincial Tumor Hospital

\section{Gaofeng Shi}

Hebei Medical University Fourth Affiliated Hospital and Hebei Provincial Tumor Hospital

\section{Jun Zhang}

Hebei Medical University Fourth Affiliated Hospital and Hebei Provincial Tumor Hospital

Zhikun Liu ( $\nabla$ zhikunliu1978@163.com )

The Fourth Hospital of Hebei Medical University

Jialiang Ren

GE Healthcare

\section{Research}

Keywords: Esophageal cancer, Radiatherapy, Enhanced CT, radiomics features, CR

Posted Date: November 3rd, 2021

DOl: https://doi.org/10.21203/rs.3.rs-998317/v1

License: (a) (1) This work is licensed under a Creative Commons Attribution 4.0 International License. Read Full License 


\section{Abstract}

Objective: Duing to chemoradiotherapy was the standard treatment for moderate and advanced esophageal cancer\tumor response to chemoradiotherapy was the most important prognostic factor.The aim of this study was to determine the value of radiomics in predicting tumor response to chemoradiotherapy in patients with esophageal squamous cell carcinoma.

Methods: 218 patients with esophageal squamous cell carcinoma diagnosed by pathology and receiving chemoradiotherapy from July 2016 to December 2017 were collected according to the ratio of 7:3,Patients were randomly divided into the training group $(n=153)$ and the validation group $(n=65)$.Clinical information of patients was collected, enhanced CT images were imported into 3D Slicer software (4.8.1 version), the radiomic features were extracted by the Python programme package.In the training group, LASSO logistic regression was performed to choose the key features and construct a radiological label, the model of predicting tumor response to chemoradiotherapy; for a better prediction, A radiomics nomogram incorporating radiological label was developed on the basis of multivariable logistic regression analysis. Model performance was determined and validated with respect to its discrimination, calibration and prediction. Clinical usefulness was estimated by decision curve analysis.

Results: According to the criteria for evaluating the curative effect of radiotherapy by esophagography combined with CT, 62 patients achieved CR, 150 patients achieved PR and 6 cases of NR, and the CR rate was 28.4\%, 27.5\% in the training group and $30.8 \%$ in the validation group $(\chi 2=0.307, p=0.858)$. The radiomics signature including seven features was significantly associated with CR after chemoradiotherapy, which constructed the radiological label.The model predicted CR after chemoradiotherapy with an area under curve (AUC) of 0.812 in the training group, and performance was similar in the validation group (AUC 0.835 ). The sensitivity, specificity and accuracy in predicting CR after chemoradiotherapy were $85.7 \%, 66.4 \%$ and $73.2 \%$, respectively. The Kappa coefficient was 0.424 ; In the validation group,the sensitivity, specificity and accuracy of were $85.0 \%, 62.2 \%$ and $69.2 \%$, respectively, and the Kappa coefficient was 0.395. The radiomics nomogram, which included the radiological label and clinical risk factors, predicted CR after chemoradiotherapy with AUC of 0.840 in the training group, and AUC 0.853 in the validation group.The sensitivity, specificity and accuracy of predicting CR were $90.5 \%, 61.3 \%, 79.3 \%$, and Kappa coefficient was 0.621 .In the validation group, the sensitivity, specificity and accuracy of predicting CR were $90.0 \%$, $54.3 \%$ and $65.6 \%$, and the Kappa coefficient was 0.643.Decision curve analysis supported that the radiomics nomogram was superior to radiological label.

Conclusion: The radiological label based on enhanced CT was a potentially tool for predicting CR after chemoradiotherapy. The radiomics nomogram model had a better prediction ability than other models and could be used as a quantitative model to predict CR after chemoradiotherapy.

\section{Introduction}

Esophageal cancer is the most common malignant tumor, with the highest incidence rate in the world. The number of newly diagnosed cases of esophageal cancer is more than 450 thousand per year(1). China is a high incidence country of esophageal cancer, and its morbidity and mortality account for more than half of the morbidity and mortality of esophageal cancer in the world(2). Most patients diagnosed with locally advanced esophageal cancers lose the opportunity for surgery at the time of diagnosis; instead, the standard treatment of concurrent chemoradiotherapy is recommended(3). However, $50-60 \%$ of patients still experienced recurrence or 
distant metastasis and died of the disease (4-5). The short-term efficacy of radical radiotherapy for esophageal cancer is significantly correlated with the long-term prognosis(6-9). Therefore, identify the patients which can not achieve complete remission before treatment, and give more active treatment such as radiotherapy and chemotherapy combined with targeted or immunotherapy, which is most likely to improve the treatment effect of patients. It is well known that accurate staging before treatment is very important for formulating individualized treatment plan, and the short-term efficacy evaluation after treatment also has a significant impact on predicting long-term survival. Due to the influence of many factors such as genetic factors, internal microenvironment, living habits and external natural environment in patients with esophageal cancer, there is obvious heterogeneity in tumors(10-11).

At present, traditional imaging methods such as gastrointestinal barium meal contrast, CT, MRI and PET were often used in clinical practice to evaluate the clinical stage and response to radiotherapy and chemotherapy of esophageal cancer. These imaging methods are mainly limited to describing the qualitative characteristics such as local morphological changes and size of tumor lesions, and there are some deviations between different observers. The internal characteristic changes of esophageal cancer may have a more important impact on the therapeutic efficacy of esophageal cancer. In order to find the characteristic changes within the tumor, it was necessary to quantitatively analyze the medical image characteristics.Radiomics can extract and analyze quantitative radiomic features from medical images to quantify tumor characteristics and function(12).

In this retrospective study, we constructed a model based on the radiomic characteristics combined with the clinical parameters to explore the relationship between the radiomic features based on enhanced CT and the short-term efficacy of patients after radiotherapy.

\section{Material And Methods}

\section{Patients}

Patients with pathologically confirmed ESCC and a Karnofsky Performance status $\geq 70$ who were receiving threedimensional conformal or intensity-modulated radiotherapy were included in this study. Patients were excluded if they met the following criteria: 1) distant metastasis; 2) low-dose palliative radiotherapy; 3) preoperative or postoperative adjuvant radiotherapy; 4) incomplete clinicopathological information; 5) esophageal fistula and esophageal stent implantation; 6) image artifacts or tumour volumes are too small to be recognised on CT images, resulting in poor visualisation quality; 7) previous malignant tumour history.

218 patients who received three-dimensional conformal or intensity-modulated radiotherapy at the Fourth Hospital of Hebei Medical University from July 2016 to December 2017 were collected. The median age was 67 years (37-84 years). All patients received electronic gastroscopy, esophageal barium meal contrast, chest enhanced CT scan and abdominal ultrasound or CT examination before treatment, according to the eighth edition of the American Joint Committee on Cancer staging criteria (13).

\section{Radiotherapy}

Gross tumour volume included the primary esophageal tumour and regional lymph nodes. The criteria for determining esophageal lesions on CT images were esophageal wall thickness $>5 \mathrm{~mm}$ or non-airless oesophagus diameter $>10 \mathrm{~mm}$, localised or whole esophageal wall thickening, and/or local lumen stenosis. The clinical target 
volume (CTV) was obtained by expanding the GTV to a margin to $2.0-3.0 \mathrm{~cm}$ at the long axis and $0.5 \mathrm{~cm}$ at the lateral axis. The planning target volume (PTV) was reached by CTV plus a margin of $0.5 \mathrm{~cm}$. The prescription dose for the whole group was 50.0-66.0 Gy, the median dose was 60.0 Gy, and a single dose was 1.8-2.2 Gy.

\section{Chemotherapy}

90 patients received 1-2 cycles of concurrent chemotherapy, with the main regimens of FP (cisplatin, 12.5 $\mathrm{mg} / \mathrm{m}^{2} \times 5$ days or $25 \mathrm{mg} / \mathrm{m}^{2} \times 3$ days; 5 -fluorouracil, 450 to $500 \mathrm{mg} / \mathrm{m}^{2} \times 5$ days) or TP (paclitaxel, $135 \mathrm{mg} / \mathrm{m}^{2}$, d1,8 days; cisplatin, $25 \mathrm{mg} / \mathrm{m}^{2}, \mathrm{~d} 2,3,4$ days, 28 days as a cycle, then 1,5 weeks of administration).

\section{CT image acquisition}

CT images were collected before and within one month after radiotherapy. All patients underwent standard chest contrast-enhanced CT scanning with a CT scanner (SOMATOM Definition Flash CT, SOMATOM Sensation Open CT, Forchheim, Germany). Scan parameters were as follows: tube voltage, 120 kV; tube current, $110 \mathrm{~mA}$; scanning matrix, 512 x 512; conventional scanning layer thickness, $5.0 \mathrm{~mm}$; reconstruction layer thickness, $1.0 \mathrm{~mm}$; mediastinal window width, $350 \mathrm{HU}$; window position, $40 \mathrm{HU}$; lung window width, $1200 \mathrm{HU}$; and window position, $-600 \mathrm{HU}$. In this study, enhanced CT images were used for tumour delineation and feature extraction.

\section{CT image segmentation}

Arterial-phase CT images of 218 patients which were retrieved from PACS were imported into the 3D Slicer software (version 4.8.1, http://www.slicer.org), The tumours were manually segmented slice by slice using the software. and an attending physician with more than 5 years of clinical experience independently outlined the region of interest $(\mathrm{ROI})$ of the esophageal primary tumours. Intraluminal air and contrast agent, fatty tissues,tumour necrosis surrounding the lesion, and blood vessels near the gross tumour were removed from the $\mathrm{ROI}$, defined as an area with attenuation values below $-50 \mathrm{HU}$ and over $300 \mathrm{HU}$. The attending physician sketched all tumour ROIs, and the associate chief physician randomly selected 40 cases of sketched tumour ROls for a consistency test.

\section{Radiomic feature extraction and selection}

Radiomic features of the segmented 3D images were extracted using the Python programme package Pyradiomics 1.2.0.(Amsterdam Netherlands). A total of seven categories of imaging features were collected in this study. This included 18 first-order, 14 shape-based histogram, 24 grey level co-occurrence matrix, 16 grey level size zone matrix, 16 grey level run length matrix, 14 grayscale dependence matrix, and 5 neighbourhood grey-tone difference matrix features (14).

The intergroup correlation coefficient (ICC) was used to analyse the consistency of the radiomic features extracted from the ROI of the tumours in the training group. The features with good reproducibility (ICC >0.75) were selected. First, Spearman correlation analysis was performed for any two feature columns. R>0.9 indicated that the two features were highly correlated, and the features with large correlation coefficients with LRFS were retained. Second, the most useful predictive features were selected using the least absolute shrinkage and selection operator (LASSO) Cox regression model, which was applied to reduce high-dimensional data. Ten-fold cross-validation was used in the parameter tuning phase of the LASSO algorithm to extract the effective and predictive features. 


\section{Construction of the radiological label and radiomics nomogram}

After the imaging features were screened by the least absolute shrinkage and selection operator (LASSO) regression, Logistic regression was used to calculate the regression coefficient ( $\beta$ ). The weighted linear formula was as follows:

rad-score $=\beta 0+\beta 1 \times 1+\beta 2 x 2+\beta 3 x 3+\ldots \ldots \ldots+\beta n X n$.

For a better prediction effect, multivariate logistic model was to build a radiomics nomogram. The nomogram was constructed by combining the radiomic signature with the conventional clinical parameters to determine the optimal predictive performance.

\section{Validation of the radiological label and radiomics nomogram}

The efficacy of the radiological label in predicting CR was determined using the receiver operating characteristic curve, area under the curve (AUC) was calculated, and the sensitivity, specificity, accuracy, positive predictive value and negative predictive value were calculated. The consistency of the two evaluation methods was defined according to the value of kappa coefficient. Decision curve analysis was then used to determine the clinical value of the constructed prediction model. In addition, we used the calibration curve to assess the predictive accuracy and the agreement between the actual and predicted CR.

\section{Response evaluation}

According to the criteria for evaluating the efficacy of radiotherapy for primary lesions of esophageal cancer proposed by our previous studies (15): $₫$ complete remission: esophagography indicates complete remission (CR), that is, the tumor disappears completely, the edge of esophageal slice is smooth, barium passes smoothly, but the esophageal wall can be slightly stiff, the lumen has no stenosis or slight stenosis, and the mucosa basically returns to normal or thickened, Chest CT showed that the maximum wall thickness of esophagus after treatment

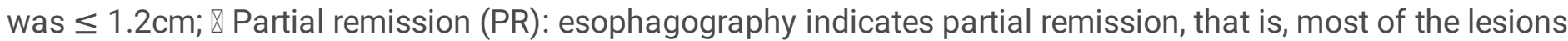
disappear without obvious distortion or angulation, no extraluminal ulcer, barium passes smoothly, but the edge is not smooth, with small filling defect and / or small niche, or although the edge is smooth, but the lumen is significantly narrow. Chest CT shows that the maximum wall thickness of the esophagus is $\leq 1.2 \mathrm{~cm}$, esophagography indicates $\mathrm{CR}$, The edge of esophageal film is smooth, barium passes smoothly, but the tube wall can be slightly stiff, the lumen is not narrow or slightly narrow, and the mucosa basically returns to normal or thickened. Chest CT shows that the maximum tube wall thickness of esophagus is $>1.2 \mathrm{~cm}$; $\mathbb{\text { No }}$ remission (NR): esophagography indicates no remission, that is, at the end of radiotherapy, there are residual lesions or no obvious improvement of lesions, but there are still obvious filling defects, niches or stenosis aggravation. Chest CT shows that the maximum tube wall thickness of esophagus is in any situation. The follow-up efficacy evaluation of this study refers to this standard.

\section{Statistical analysis}

Statistical analysis was performed using R software (version 3.4.4) and SPSS version 25.0 (IBM). Comparisons of patient characteristics were performed using X2 test or two-sample t-test. Univariate analysis used X2 test to compare the $\mathrm{CR}$ of different groups of esophageal cancer patients. Logistic regression model was used to screen the independent influencing factors of CR. P $<0.05$ was statistically significant. 


\section{Results}

\section{Clinical characteristics of patients}

Of the 218 patients included in the study, median age was 67.0(37.0-84.0) years. They were randomly divided into training group and validation group at a ratio of 7:3. There were no significant statistical differences between the two groups in age, gender, lesion site, lesion length, maximum layer tube wall thickness of esophageal lesion, local external invasion, supraclavicular lymph node metastasis, TNM stage, chemotherapy or not, concurrent or not, radiotherapy dose $(p>0.05)$ (Table 1$)$. 
Table 1

Clinical characteristics of patients with ESCC in the training cohort and validation cohort

\begin{tabular}{|c|c|c|c|c|}
\hline Factors & $\begin{array}{l}\text { Training cohort } \\
(n=153)\end{array}$ & $\begin{array}{l}\text { Validation cohort } \\
(\mathrm{n}=65)\end{array}$ & $x^{2} / t$ & $\mathbf{P}$ \\
\hline Age (years) & $66.88 \pm 8.86$ & $65.42 \pm 8.10$ & 1.141 & 0.255 \\
\hline \multicolumn{5}{|l|}{ Gender } \\
\hline Male & 103 & 46 & \multirow[t]{2}{*}{0.251} & \multirow[t]{2}{*}{0.616} \\
\hline Female & 50 & 19 & & \\
\hline \multicolumn{5}{|l|}{ Location } \\
\hline Cervical & 8 & 3 & \multirow[t]{4}{*}{0.288} & \multirow[t]{4}{*}{0.966} \\
\hline Upper & 40 & 17 & & \\
\hline Middle & 81 & 33 & & \\
\hline Lower & 24 & 12 & & \\
\hline Length (cm) & $5.30 \pm 1.997$ & $5.41 \pm 2.160$ & 0.382 & 0.703 \\
\hline $\begin{array}{l}\text { Maximum tumour wall thicknesses (Pre- } \\
\text { RT) (cm) }\end{array}$ & $1.457 \pm 0.471$ & $1.380 \pm 0.426$ & 1.146 & 0.253 \\
\hline \multicolumn{5}{|l|}{ T stage } \\
\hline T1-3 & 115 & 47 & \multirow[t]{2}{*}{0.915} & \multirow[t]{2}{*}{0.659} \\
\hline T4 & 38 & 18 & & \\
\hline \multicolumn{5}{|l|}{ Tracheal invasion } \\
\hline No & 119 & 51 & \multirow[t]{2}{*}{0.012} & \multirow[t]{2}{*}{0.911} \\
\hline Yes & 34 & 14 & & \\
\hline \multicolumn{5}{|l|}{ Prevertebral soft tissue invasion } \\
\hline No & 143 & 59 & 0.171 & 0.679 \\
\hline Yes & 10 & 6 & & \\
\hline \multicolumn{5}{|l|}{ Aortic invasion } \\
\hline No & 140 & 59 & \multirow[t]{2}{*}{0.039} & \multirow[t]{2}{*}{0.844} \\
\hline Yes & 13 & 6 & & \\
\hline \multicolumn{5}{|l|}{ Supraclavicular lymph node metastasis } \\
\hline Yes & 118 & 54 & \multirow[t]{2}{*}{0.971} & \multirow[t]{2}{*}{0.324} \\
\hline No & 35 & 11 & & \\
\hline \multicolumn{5}{|l|}{ N stage } \\
\hline NO & 11 & 10 & 4.856 & 0.183 \\
\hline
\end{tabular}




\begin{tabular}{|c|c|c|c|c|}
\hline Factors & $\begin{array}{l}\text { Training cohort } \\
(n=153)\end{array}$ & $\begin{array}{l}\text { Validation cohort } \\
(\mathrm{n}=65)\end{array}$ & $x^{2} / t$ & $\mathbf{P}$ \\
\hline N1 & 54 & 16 & & \\
\hline N2 & 58 & 26 & & \\
\hline N3 & 30 & 13 & & \\
\hline \multicolumn{5}{|c|}{ TNM stage } \\
\hline$\Delta-\mathbb{Z}$ & 95 & 39 & \multirow[t]{2}{*}{0.084} & \multirow[t]{2}{*}{0.772} \\
\hline 『a & 58 & 26 & & \\
\hline \multicolumn{5}{|c|}{ Chemotherapy } \\
\hline Yes & 89 & 43 & \multirow[t]{2}{*}{1.217} & \multirow[t]{2}{*}{0.270} \\
\hline No & 64 & 22 & & \\
\hline \multicolumn{5}{|c|}{ Chemoradiotherapy } \\
\hline Yes & 62 & 28 & \multirow[t]{2}{*}{0.276} & \multirow[t]{2}{*}{0.599} \\
\hline No & 27 & 15 & & \\
\hline \multicolumn{5}{|c|}{ Radiation dose (Gy) } \\
\hline$<60$ & 53 & 22 & 0.053 & 0.974 \\
\hline 60 & 46 & 19 & & \\
\hline$>60$ & 54 & 24 & & \\
\hline
\end{tabular}

\section{Short-term efficacy of primary lesion after radiotherapy}

According to the criteria of esophageal barium meal imaging, 69 patients achieved CR, 143 patients achieved PR and 6 patients achieved NR after radiotherapy, and the CR rate was $31.7 \%$, including $30.7 \%$ in the training group and $33.8 \%$ in the validation group. There was no difference between the two groups $(X 2=0.266, P=0.875)$. In the whole group, the maximum wall thickness of esophageal lesions was $1.45 \pm 0.45 \mathrm{~cm}$ before treatment and $1.10 \pm 0.41 \mathrm{~cm}$ after treatment, among which 145 cases $(66.5 \%)$ and 73 cases $(33.5 \%)$ of the maximum wall thickness of esophageal lesions $\leq 1.2 \mathrm{~cm}$ after treatment. According to the evaluation criteria of the short-term efficacy of esophageal barium meal angiography combined with CT, 62 patients achieved CR, 150 patients achieved PR and 6 cases of NR, and the CR rate was $28.4 \%, 27.5 \%$ in the training group and $30.8 \%$ in the validation group. There was no significant difference between the two groups $(X 2=0.307, P=0.858)$.

\section{Relationship between clinical factors and short-term efficacy of primary lesion after radiotherapy for esophageal cancer}

In the training group, the relationship between clinical factors of patients and the short-term efficacy of the primary lesion after radiotherapy was analyzed. Univariate analysis showed that the maximum layer tube wall thickness of esophageal lesions before radiotherapy, trachea invasion, TNM stage were closely related to the 
short-term efficacy of the primary lesion $(p<0.05)$ (Table 2$)$. Multivariate regression analysis showed that the tube wall thickness at the maximum layer of esophageal lesions before radiotherapy was an independent factor affecting the short-term efficacy of the primary lesion in patients with esophageal cancer, and the optimal value was $1.19 \mathrm{~cm}$. AUC for CR status prediction was $0.801(95 \% \mathrm{Cl} 0.688-0.913)$ and $0.789(95 \% \mathrm{Cl} 0.713-0.865)$ in the training group and the validation group. 
Table 2

Relationship between clinical characteristics and short-term outcome after radiotherapy in patients with esophageal cancer

\begin{tabular}{|c|c|c|c|c|c|c|}
\hline \multirow[t]{2}{*}{ Factors } & \multicolumn{2}{|c|}{$\begin{array}{l}\text { Training cohort } \\
(n=153)\end{array}$} & \multirow[t]{2}{*}{$\mathbf{P}$} & \multicolumn{2}{|c|}{$\begin{array}{l}\text { Validation cohort } \\
(n=65)\end{array}$} & \multirow[t]{2}{*}{$\mathbf{P}$} \\
\hline & CR & Non-CR & & CR & Non-CR & \\
\hline \multicolumn{7}{|l|}{ Gender } \\
\hline Male & 27 & 76 & \multirow[t]{2}{*}{0.623} & 13 & 33 & \multirow[t]{2}{*}{0.495} \\
\hline Female & 15 & 35 & & 7 & 12 & \\
\hline \multicolumn{7}{|l|}{ Location } \\
\hline Cervical & 2 & 6 & \multirow[t]{4}{*}{0.881} & 2 & 1 & \multirow[t]{4}{*}{0.444} \\
\hline Upper & 13 & 27 & & 6 & 11 & \\
\hline Middle & 20 & 61 & & 8 & 25 & \\
\hline Lower & 7 & 17 & & 4 & 8 & \\
\hline \multicolumn{7}{|c|}{ Lesion length } \\
\hline$\leq 5 \mathrm{~cm}$ & 25 & 55 & \multirow[t]{2}{*}{0.270} & 11 & 22 & \multirow[t]{2}{*}{0.649} \\
\hline$>5 \mathrm{~cm}$ & 17 & 56 & & 9 & 23 & \\
\hline \multicolumn{7}{|c|}{$\begin{array}{l}\text { Maximum tumour wall thicknesses (Pre- } \\
\text { RT) }\end{array}$} \\
\hline$\leq 1.5 \mathrm{~cm}$ & 32 & 45 & \multirow[t]{2}{*}{0.000} & 12 & 20 & \multirow[t]{2}{*}{0.247} \\
\hline$>1.5 \mathrm{~cm}$ & 10 & 66 & & 8 & 25 & \\
\hline \multicolumn{7}{|l|}{ T stage } \\
\hline T1-3 & 38 & 77 & \multirow[t]{2}{*}{0.007} & 19 & 28 & \multirow[t]{2}{*}{0.006} \\
\hline T4 & 4 & 34 & & 1 & 17 & \\
\hline \multicolumn{7}{|c|}{ Tracheal invasion } \\
\hline No & 38 & 81 & \multirow[t]{2}{*}{0.020} & 18 & 33 & \multirow[t]{2}{*}{0.131} \\
\hline Yes & 4 & 30 & & 2 & 12 & \\
\hline \multicolumn{7}{|c|}{ Prevertebral soft tissue invasion } \\
\hline No & 41 & 102 & \multirow[t]{2}{*}{0.201} & 20 & 39 & \multirow[t]{2}{*}{0.087} \\
\hline Yes & 1 & 9 & & 0 & 6 & \\
\hline \multicolumn{7}{|c|}{ Aortic invasion } \\
\hline No & 40 & 100 & \multirow[t]{2}{*}{0.308} & 20 & 40 & \multirow[t]{2}{*}{0.121} \\
\hline Yes & 2 & 11 & & 0 & 5 & \\
\hline
\end{tabular}




\begin{tabular}{|c|c|c|c|c|c|c|}
\hline \multirow[t]{2}{*}{ Factors } & \multicolumn{2}{|c|}{$\begin{array}{l}\text { Training cohort } \\
(n=153)\end{array}$} & \multirow[t]{2}{*}{$\mathbf{P}$} & \multicolumn{2}{|c|}{$\begin{array}{l}\text { Validation cohort } \\
(n=65)\end{array}$} & \multirow[t]{2}{*}{$\mathbf{P}$} \\
\hline & CR & Non-CR & & CR & Non-CR & \\
\hline \multicolumn{7}{|c|}{ Supraclavicular lymph node metastasis } \\
\hline Yes & 11 & 24 & \multirow[t]{2}{*}{0.548} & 4 & 7 & \multirow[t]{2}{*}{0.659} \\
\hline No & 31 & 87 & & 16 & 38 & \\
\hline \multicolumn{7}{|l|}{ N stage } \\
\hline NO & 7 & 4 & \multirow[t]{4}{*}{0.030} & 6 & 4 & \multirow[t]{4}{*}{0.231} \\
\hline N1 & 11 & 43 & & 4 & 12 & \\
\hline N2 & 17 & 41 & & 7 & 19 & \\
\hline N3 & 7 & 23 & & 3 & 10 & \\
\hline \multicolumn{7}{|l|}{ TNM stage } \\
\hline$\nabla-\rrbracket$ & 32 & 63 & \multirow[t]{2}{*}{0.027} & 17 & 22 & \multirow[t]{2}{*}{0.006} \\
\hline$\llbracket a$ & 10 & 48 & & 3 & 23 & \\
\hline \multicolumn{7}{|c|}{ Albumin level (Pre-RT) } \\
\hline High $(\geq 40)$ & 20 & 61 & \multirow[t]{2}{*}{0.208} & 5 & 25 & \multirow[t]{2}{*}{0.048} \\
\hline Low $(<40)$ & 22 & 50 & & 15 & 20 & \\
\hline \multicolumn{7}{|c|}{ Chemotherapy } \\
\hline Yes & 20 & 69 & \multirow[t]{2}{*}{0.104} & 16 & 27 & \multirow[t]{2}{*}{0.116} \\
\hline No & 22 & 42 & & 4 & 18 & \\
\hline \multicolumn{7}{|c|}{ Chemoradiotherapy } \\
\hline Yes & 17 & 45 & \multirow[t]{2}{*}{0.090} & 11 & 17 & \multirow[t]{2}{*}{0.700} \\
\hline No & 3 & 24 & & 5 & 10 & \\
\hline \multicolumn{7}{|c|}{ Radiation dose(Gy) } \\
\hline$<60$ & 16 & 37 & \multirow[t]{3}{*}{0.354} & 7 & 15 & \multirow[t]{3}{*}{0.506} \\
\hline 60 & 9 & 37 & & 4 & 15 & \\
\hline$>60$ & 17 & 37 & & 9 & 15 & \\
\hline
\end{tabular}

Selection of radiomic features and building a radiomics label for predicting short-term efficacy of primary lesion after radiotherapy for esophageal cancer 
Before features selection, 1037 radiomic features extracted from the CT images. Using the filter criteria of ICCs $\geq 0.900$, we derived a list of 654 features (ICC range:0.900-1.000). Next, we used the LASSO algorithm to select the most significant radiomics features for classifying CR and Non-CR patients in the training set (Fig. 1). The result showed that seven features were identified.A radiological label was established to predict short-term efficacy of primary lesion in patients with esophageal cancer after radiotherapy. The formula was expressed as follows:

Rad-score $=1.277-0.698$ * Bef_original_shape_Least Axis Length+ 0.577 * Bef_original_ngtdm_Busyness

-0.403 * Bef_original_gldm_SmallDependenceLowGrayLevelEmphasis $₫ 0.528$ *

Aft_wavelet.LLH_glcm_Correlation+

0.400 * Aft_wavelet.LHL_glszm_Gray Level NonUniformityNormalized+ 0.827 * Aft_original_shape_Least Axis Length

- 0.458 * Aft_original_first order_Median

\section{Validating of the radiomics label}

The AUC of radiological label for predicting CR evaluated by ROC curve was $0.812(95 \% \mathrm{Cl} 0.739-0.886)$ and $0.835(95 \% \mathrm{Cl} 0.730-0.940)$ in the training group and the validation group(Fig. 2). According to the optimal cut-off point which was 0.72 , patients were divided into high probability group and low probability group with $\mathrm{CR}$. In the training group, the sensitivity, specificity and accuracy of predicting CR were $85.7 \%, 66.4 \%$ and $73.2 \%$, and the Kappa coefficient was 0.424 . In the validation group, the sensitivity, specificity and accuracy of predicting the short-term efficacy after radiotherapy for esophageal cancer were $85.0 \%, 62.2 \%$ and $69.2 \%$, and the Kappa value was 0.395 (Table 3 ).

Table 3

Predictive performance of discrimination of all models

\begin{tabular}{|llllllll|}
\hline Group & Model & sensitivity(\%) & specificity(\%) & accuracy(\%) & $\begin{array}{l}\text { Positive } \\
\text { predictive } \\
\text { value(\%) }\end{array}$ & $\begin{array}{l}\text { Negative } \\
\text { predictive } \\
\text { value(\%) }\end{array}$ & Kappa \\
\hline $\begin{array}{l}\text { Training } \\
\text { cohort }\end{array}$ & $\begin{array}{l}\text { Clinical } \\
\text { character }\end{array}$ & 69.0 & 68.5 & 68.6 & 45.3 & 85.4 & 0.323 \\
& $\begin{array}{l}\text { ladiomics } \\
\text { label }\end{array}$ & 85.7 & 66.4 & 73.2 & 49.3 & 92.4 & 0.424 \\
\hline $\begin{array}{l}\text { radiomics } \\
\text { nomogram }\end{array}$ & 90.5 & 61.3 & 79.3 & 46.9 & 94.4 & 0.621 \\
\hline $\begin{array}{l}\text { Validation } \\
\text { cohort }\end{array}$ & $\begin{array}{l}\text { Clinical } \\
\text { character }\end{array}$ & 55.0 & 75.0 & 63.1 & 42.3 & 76.9 & 0.200 \\
\hline $\begin{array}{l}\text { radiomics } \\
\text { label }\end{array}$ & 85.0 & 62.2 & 69.2 & 50.0 & 90.3 & 0.395 \\
\hline $\begin{array}{l}\text { radiomics } \\
\text { nomogram }\end{array}$ & 90.0 & 54.3 & 65.6 & 46.2 & 92.3 & 0.643 \\
\hline
\end{tabular}




\section{Construction of the radiomics nomogram}

The results of univariate analysis showed that: maximum layer tube wall thickness of esophageal lesions before radiotherapy, trachea invasion, T stage, $\mathrm{N}$ stage, TNM stage and radiomics label were the factors related to $\mathrm{CR}(\mathrm{P}<0.05)$. Multivariate logistic analysis showed that $\mathrm{T}$ stage and radiological label were independent factors affecting the short-term efficacy of radiotherapy (Figure $3 \mathrm{~A}$ ). T stage and radiological label were combined to construct a radiomics nomogram model for predicting $\mathrm{CR}$ after radiotherapy, and the model $=-2.583+0.823 * \mathrm{~T}$ staging $+4.961 *$ radiological label.

\section{Validating of the radiomics nomogram}

The AUC of radiomics nomogram for predicting CR evaluated by ROC curve was $0.840(0.775-0.906)$ and $0.853(0.755-0.952)$ in the training group and the validation group(Figure 2$)$. The patients was divided into the high probability group and the low probability group with CR by the optimal cut-off point(0.84). In the training group, the sensitivity, specificity and accuracy of predicting CR were $90.5 \%, 61.3 \%$ and $79.3 \%$, and the Kappa coefficient was 0.621 . In the validation group, the sensitivity, specificity and accuracy of predicting the short-term efficacy after radiotherapy for esophageal cancer were $90.0 \%, 54.3 \%$ and $65.6 \%$, and the Kappa value was 0.643(Table

3). The calibration curve showed that there was no significant statistical difference between the predicted value by the model and the observed values(p凶0.05) (Figure 3B, C). Decision curve analysis (DCA) suggested that radiomics nomogram was superior to clinical model and radiomics label in predicting $\mathrm{CR}$ after radiotherapy for esophageal cancer (Figure 3D).

\section{Discussion}

Esophageal cancer is one of the most common malignant tumors of digestive system. Chemoradiotherapy has become the standard treatment method for patients with ESCC who lose the opportunity for operation or refused surgery[11], Patients who achieved complete remission (CR) after chemoradiotherapy had a better prognosis than those who did not(16). Therefore, establishing a model to predict the response of esophageal cancer to chemoradiotherapy will help us to pre-classify the radiotherapy efficacy of patients, formulate individualized treatment plans before treatment, and achieve the purpose of accurate treatment.

Radiomics is a new image analysis method, which can convert CT, MRI and PET-CT images into image feature data, including intensity, texture, shape, wavelet features(17), to achieve a comprehensive quantitative description of tumor diagnosis, efficacy prediction and survival prognosis analysis(18). At present, several clinical studies mainly focus on predicting treatment response and prognosis(19-25). For example, Hou extracted 214 radiomics features from the enhanced CT images of 49 patients with esophageal cancer, and screened out the prediction model established by 5 radiomics features. The AUC was 0.727 and the accuracy rate was 0.891 in predicting CR status of esophageal cancer patients treated with CCRT. Jin established a model to predict the response of esophageal cancer to radiotherapy and chemotherapy in combination with CT imaging characteristics and dosimetric parameters. Unfortunately, the sample size of patients included in the study was small.

In this retrospective study, The regions of interest of the tumor of 218 patients with esophageal cancer received radiotherapy were delineated from the enhanced CT images of esophageal cancer patients before and after radiotherapy. The radiomics features, extracted from CT images, with strong correlation were removed and reduced in dimension by using the methods of correlation analysis and Lasso, Logistic regression was used to 
screen the radiomics features closely related to the short-term efficacy of radiotherapy. A prediction model for predicting the short-term efficacy of radiotherapy was constructed with the selecting seven radiomics features. The AUC value of the model in the training group was 0.812 and that in the validation group was 0.835 . The sensitivity, specificity and accuracy of predicting the efficacy of radiotherapy in the training group were $95.7 \%$ and $66.4 \% 73.2 \%, 85.0 \%, 62.2 \%$ and $69.2 \%$ in the validation group. The model provide an economical and noninvasive method for clinicians to predict the treatment response of esophageal cancer patients receiving chemoradiotherapy.

As well known, tumor stage is the most important prognostic factor for patients with malignant tumors. Clinical research showed that patients with late $\mathrm{T}$ stage before treatment have a lower probability of achieving $\mathrm{CR}$ after chemoradiotherapy(26), which was consistent with our research results. We analyzed the data of 153 patients in the training group and found that the rate of $\mathrm{CR}$ in T4 stage patients after radiotherapy was $10.5 \%$, much lower than the $\mathrm{CR}$ rate in $\mathrm{T} 1-3$ patients(33.0\%) $(\mathrm{P}<0.05)$. The results confirmed that there was a significant correlation between the $T$ stage and the $\mathrm{CR}$ rate after radiotherapy. However, we also found that even patients with early $\mathrm{T}$ stage achieved only $33.0 \% \mathrm{CR}$ rate after radiotherapy, because the T-stage in the current staging standard is based on the depth of esophageal tumor invasion into the esophageal tube wall and its relationship with surrounding tissues and organs. The representative infiltration depth is only the horizontal axis direction of esophageal cancer invasion, not the longitudinal axis direction of esophageal cancer invasion(27). Therefore, the current TNM staging system lacks sufficient information for a comprehensive evaluation of primary tumors. At the same time, the clinical staging of esophageal cancer depends too much on imaging examination, which leads to inconsistency and even great difference between imaging staging and pathological staging.

Radiomics can extract the image information of tumor and provide more comprehensive information representing tumor, so as to improve the accuracy of clinical staging. Our results showed that there was a significant correlation between the clinical stage before treatment and the short-term efficacy after radiotherapy, which was statistically significant in both the training group and the validation group $(P<0.05)$. The multivariate analysis of our study showed that the T-stage and radiological label before treatment were independent factors affecting the short-term efficacy of esophageal cancer after radiotherapy. Therefore, an radiomics nomogram model combining clinical and imaging characteristics was constructed. AUC of ROC curve was 0.840 in the training group and 0.853 in the validation group. The sensitivity, specificity and accuracy of predicting radiotherapy efficacy in the training group were $90.5 \%, 61.3 \%$ and $79.3 \%$, and $90.0 \%$ and $54.3 \%$ in the validation group $65.6 \%$, which was better than the simple clinical feature model and radiology label. It may be an effective tool to predict the shortterm efficacy of esophageal cancer patients for chemoradiotherapy.

Of course, there are inevitably some defects or deficiencies in our study: first, It was a retrospective single center survey. There were more or less biases or deficiencies in the process of clinical data collection, which may have impact on the study.second, The sample size of the study was too small and the stability of the results was affected. The radiomics nomogram model was only verified in a validation group with a small sample size. The conclusion of the study also needed to be repeatedly verified internally and externally in a larger patient sample population, so that the model can be repaired and mature continuously. Third, The short-term efficacy evaluation of radiotherapy in this study failed to carry out pathological evaluation, which was evaluated using CT and esophageal barium radiography. Although our hospital has done a lot of research work in the evaluation standard of radiotherapy efficacy for esophageal cancer. The proposed standard has been recognized by domestic peers and has gradually become the standard for judging the short-term efficacy of radiotherapy for primary lesions of

Page 14/20 
esophageal cancer. However, there was a certain deviation from the gold standard of pathological evaluation, which may affect the accuracy of the results. Fourth, radiomics has some difficulties in practical operation, such as: image quantitative processing, image segmentation. Most of the ROI delineation methods of esophageal cancer used manual delineation. Although the accuracy of manual segmentation is higher than that of semiautomatic segmentation, there were subjective differences in the delineation of lesions, especially the determination of the junction between tumor and normal esophageal tissue.

Although there were some deficiencies in the application of radiomics, we established a more comprehensive prediction model for the efficacy of esophageal cancer radiotherapy by retrospectively analyzing the imaging characteristics of esophageal cancer patients, combined with clinical factors, so as to help doctors to formulate individualized diagnosis and treatment plans.

\section{Abbreviations}

LASSO: Least absolute shrinkage and selection operator; CR: Complete remission; PR: Partial remission; NR: No remission; AUC: Area under the curve; ROC: Receiver operating characteristic curve; CT: Computed tomography; MRI: Magnetic resonance imaging; PET:Positron emission tomography; ESCC: Esophageal squamous cell cancerl; GTV: Gross tumour volume; CTV: Clinical target volume; PTV: Planning target volume; ROI: Region of interest; ICC: Intergroup correlation coefficient; DCA: Decision curve analysis; ICC: Intergroup correlation coefficient.

\section{Declarations}

\section{Acknowledgments}

Not applicable.

\section{Author Contributions}

JK, ZL and SZ designed the study. JK and ZL accomplished the manuscript. JK and LC collected the required CT data. JK and JZ evaluated the curative effect of radiotherapy for esophageal cancer and determined the local control status of oesophagea cancer. LC collected and assembled the clinical data. JR performed the statistical analysis and interpretation. JK determined the selection of references and experimental standards. JK and GS performed the data analysis and interpretation. SZ performed manuscript approval and modification. All authors contributed to the article and approved the submitted version.

\section{Funding}

This study was supported by the National Natural Science Foundation of China(Grant No. 81872456) and the Health Innovation Special Fund of Hebei Province (Grant No. 213777104D).

\section{Availability of data and materials}

All data generated or analyzed during this study are included in this manuscript and its Additional files.

\section{Ethics approval and consent to participate}


The studies involving human participants were reviewed and approved by ethics committee of the Fourth Hospital of Hebei Medical University. Written informed consent was not required, due to the retrospective nature of the study.

\section{Consent for publication}

Not applicable.

\section{Conflict of Interest}

JR was employed by GE Healthcare China.The remaining authors declare that the research was conducted in the absence of any commercial or financial relationships that could be construed as a potential conflict of interest.

\section{References}

1. Pennathur A M, Gibson M K M, Jobe B A P, et al. esophageal carcinoma. The Lancet (British edition), 2013,381(9864):400-412.

2. Ferlay J, Soerjomataram I, Dikshit R, et al. Cancer incidence and mortality worldwide: sources, methods and major patterns in GLOBOCAN 2012. Int J Cancer, 2015,136(5):E359-E386.

3. Lagergren J, Smyth E, Cunningham D, et al. esophageal cancer. Lancet, 2017,390(10110):2383-2396.

4. Minsky B D, Pajak T F, Ginsberg R J, et al. INT 0123 (Radiation Therapy Oncology Group 94-05) phase III trial of combined-modality therapy for esophageal cancer: high-dose versus standard-dose radiation therapy. $\mathrm{J}$ Clin Oncol, 2002,20(5):1167-1174.

5. Welsh J, Settle S H, Amini A, et al. Failure patterns in patients with esophageal cancer treated with definitive chemoradiation. Cancer, 2012,118(10):2632-2640.

6. Stahl M, Stuschke M, Lehmann N, et al. Chemoradiation with and without surgery in patients with locally advanced squamous cell carcinoma of the esophagus. J Clin Oncol, 2005,23(10):2310-2317.

7. Nakajo M, Jinguji M, Nakabeppu Y, et al. Texture analysis of 18F-FDG PET/CT to predict tumour response and prognosis of patients with esophageal cancer treated by chemoradiotherapy. European Journal of Nuclear Medicine and Molecular Imaging, 2017,44(2):206-214.

8. van Hagen P, Hulshof M C, van Lanschot J J, et al. Preoperative chemoradiotherapy for esophageal or junctional cancer. N Engl J Med, 2012,366(22):2074-2084.

9. Liu S, Xi M, Yang H, et al. Is There a Correlation Between Clinical Complete Response and Pathological Complete Response After Neoadjuvant Chemoradiotherapy for Esophageal Squamous Cell Cancer?. Annals of Surgical Oncology, 2016,23(1):273-281.

10. Baba Y, Watanabe M, Baba H. Review of the alterations in DNA methylation in esophageal squamous cell carcinoma. Surg Today, 2013,43(12):1355-1364.

11. Islami F, Cao Y, Kamangar F, et al. Reproductive factors and risk of esophageal squamous cell carcinoma in northern Iran: a case-control study in a high-risk area and literature review. Eur J Cancer Prev, 2013,22(5):461466.

12. Kumar V, Gu Y, Basu S, et al. Radiomics: the process and the challenges. Magn Reson Imaging, 2012,30(9):1234-1248. 
13. Thomas W Rice, Hemant Ishwaran, Eugene H Blackstone, et al. Recommendations for clinical staging (cTNM) of cancer of the esophagus and esophagogastric junction for the 8th edition AJCC/UICC staging manuals.Dis Esophagus(2016 )29:913-919.

14. Umeoka S, Koyama T, Togashi K, et al. Esophageal cancer: evaluation with triple-phase dynamic CT--initial experience. Radiology, 2006,239(3): 777-783.

15. Ren Xuejiao, Wang Lan, Chen Liying, et al. Clinical application of barium radiography and computed tomography-based short-term outcome evaluation criteria in esophageal cancer. Chin J Radiat Oncol, 2018,27(5): 449-454.

16. Liu SL, Xi M, Yang H, et al. Is there a correlation between clinical complete response and pathological complete response after neoadjuvant chemoradiotherapy for esophageal squamous cell cancer? Ann Surg Oncol. 2016;23(1):273-81.

17. Lambin P, Rios-Velazquez E, Leijenaar R, et al. Radiomics: Extracting more information from medical images using advanced feature analysis. European Journal of Cancer, 2012,48(4):441-446.

18. Gillies R J, Kinahan P E, Hricak H. Radiomics: Images Are More than Pictures, They Are Data. Radiology, 2016,278(2):563-577.

19. Yang Z, He B, Zhuang X, et al. CT-based radiomic signatures for prediction of pathologic complete response in esophageal squamous cell carcinoma after neoadjuvant chemoradiotherapy. JOURNAL OF RADIATION RESEARCH, 2019,60(4):538-545.

20. Yip C, Landau D, Kozarski R, et al. Primary esophageal cancer: heterogeneity as potential prognostic biomarker in patients treated with definitive chemotherapy and radiation therapy. Radiology, 2014,270(1):141148.

21. Jin X, Zheng X, Chen D, et al. Prediction of response after chemoradiation for esophageal cancer using a combination of dosimetry and CT radiomics. EUROPEAN RADIOLOGY, 2019,29(11):6080-6088.

22. Ganeshan B, Skogen K, Pressney I, et al. Tumour heterogeneity in esophageal cancer assessed by CT texture analysis: Preliminary evidence of an association with tumour metabolism, stage, and survival. Clinical Radiology, 2012,67(2):157-164.

23. Hou Z, Li S, Ren W, et al. Radiomic analysis in T2W and SPAIR T2W MRI: predict treatment response to chemoradiotherapy in esophageal squamous cell carcinoma. Journal of Thoracic Disease, 2018,10(4):22562267.

24. Larue R T H M, Klaassen R, Jochems A, et al. Pre-treatment CT radiomics to predict 3-year overall survival following chemoradiotherapy of esophageal cancer. ACTA ONCOLOGICA, 2018,57(11):1475-1481.

25. Hou Z, Ren W, Li S, et al. Radiomic analysis in contrast-enhanced CT: predict treatment response to chemoradiotherapy in esophageal carcinoma. ONCOTARGET, 2017,8(61):104444-104454.

26. Blum M M, Xiao L, Patel V R, et al. Pathological complete response in patients with esophageal cancer after the trimodality approach: The association with baseline variables and survival-The University of Texas MD Anderson Cancer Center experience. Cancer, 2017,123(21):4106-4113.

27. Rice T W, Ishwaran H, Hofstetter W L, et al. Recommendations for pathologic staging (pTNM) of cancer of the esophagus and esophagogastric junction for the 8th edition AJCC/UICC staging manuals. Dis Esophagus, 2016,29(8):897-905.

\section{Figures}

Page $17 / 20$ 
$\begin{array}{lllllllllllll}78 & 77 & 76 & 73 & 73 & 69 & 62 & 50 & 39 & 25 & 17 & 11 & 2\end{array}$

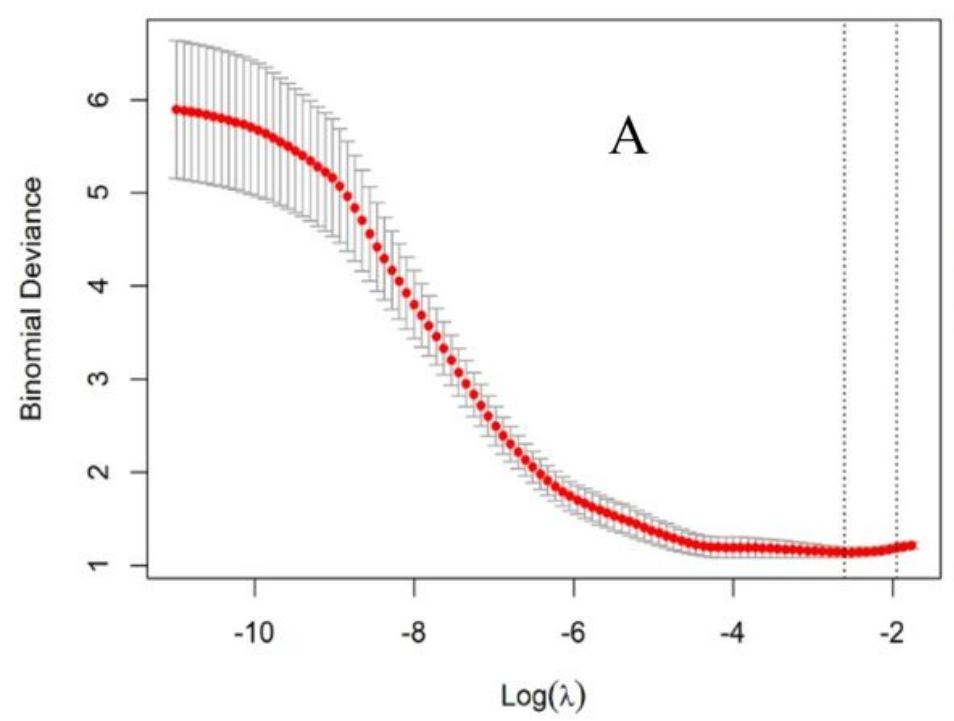

$\begin{array}{llllllllllllll}67 & 67 & 67 & 65 & 63 & 57 & 51 & 38 & 33 & 19 & 13 & 6 & 2 & 0\end{array}$

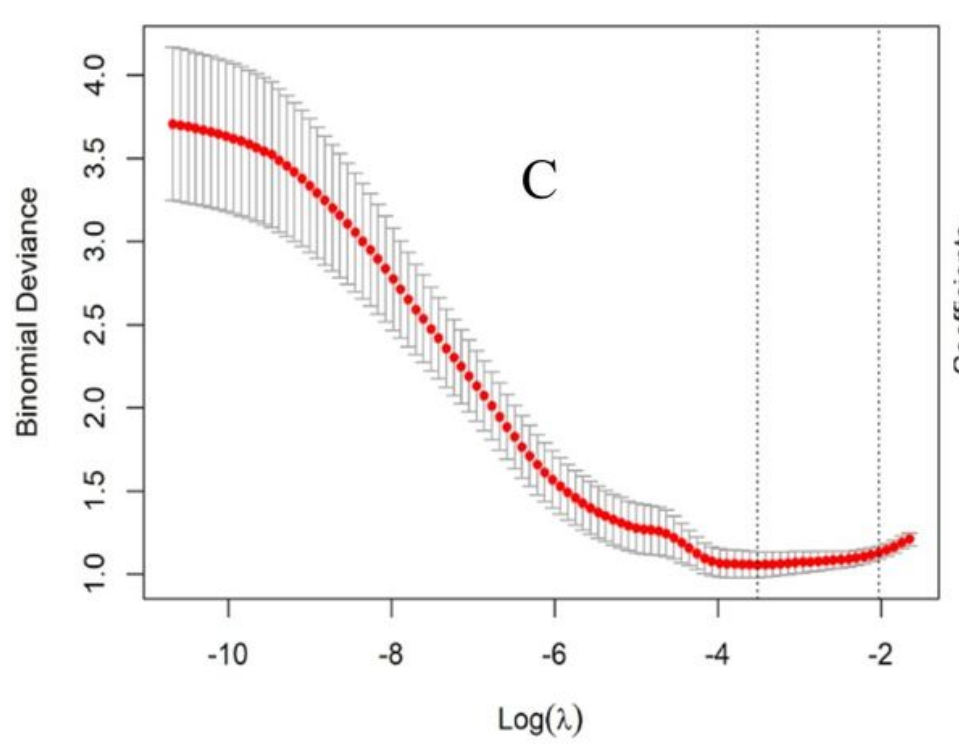

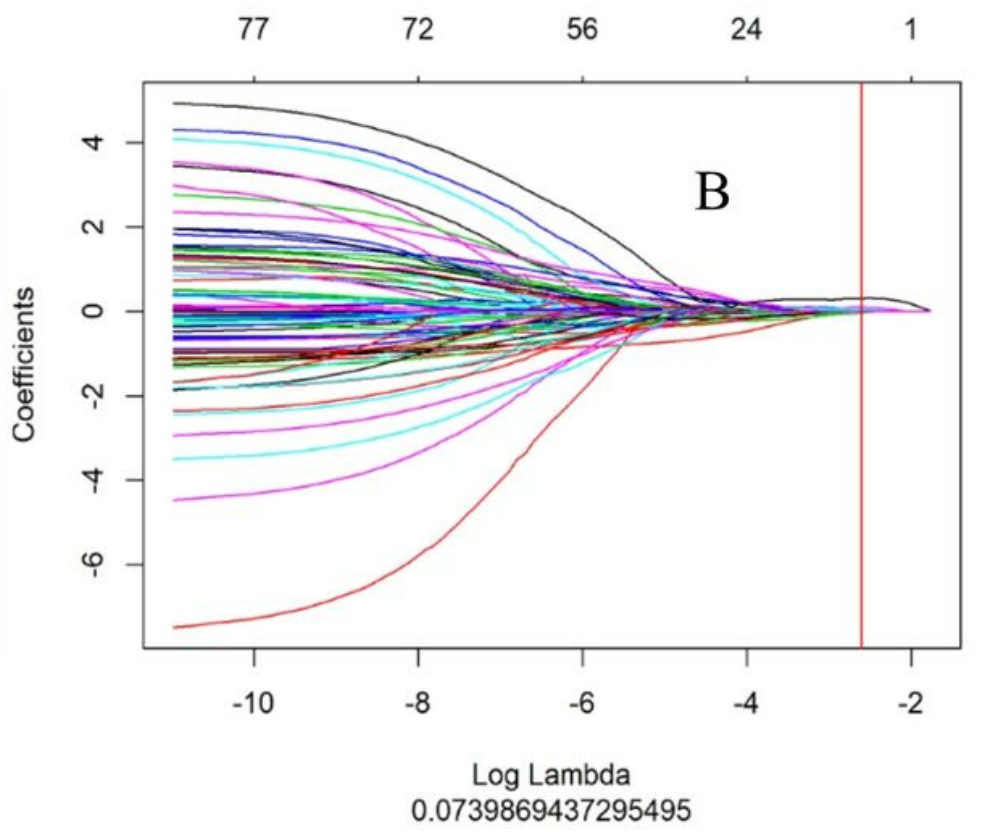

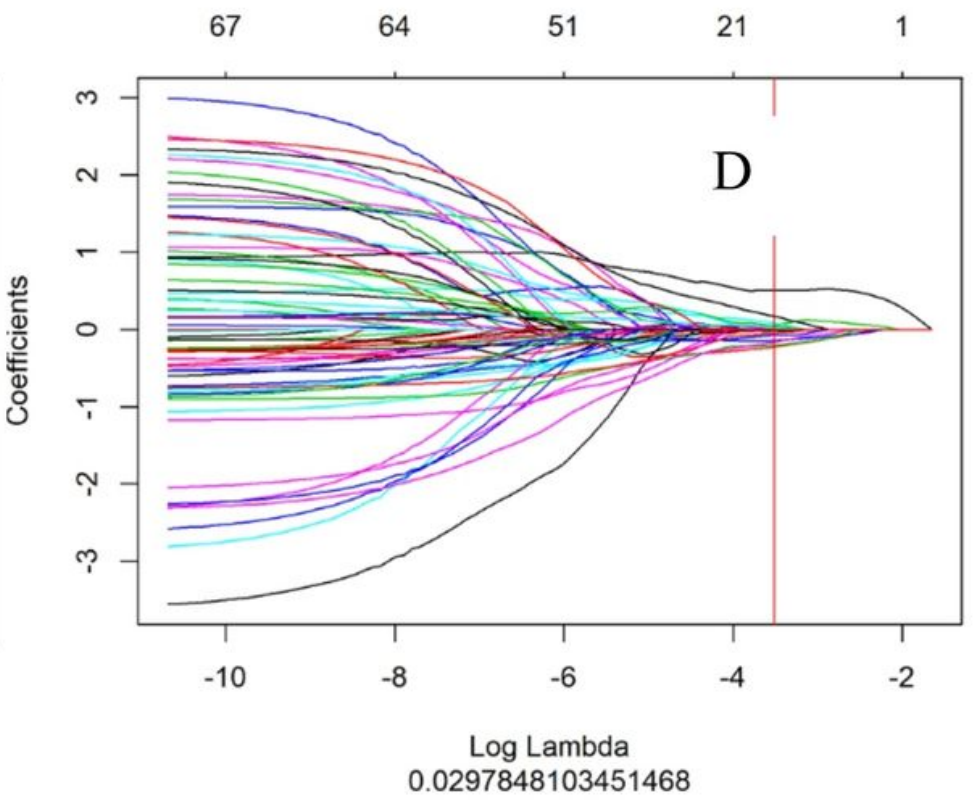

\section{Figure 1}

The least absolute shrinkage and selection operator (Lasso) regression model was used to select the radiomics feature before and after radiotherapy. A Turning penalization parameter lambda $(\lambda)$ using 10 -fold crossvalidation and minimum criterion in Lasso model『Pre-RT区; B Lasso coefficient profiles of the 1037 radiomics features. The vertical gray line was drawn at the value selected using 10-fold cross-validation囚Pre-RT囚; C Turning penalization

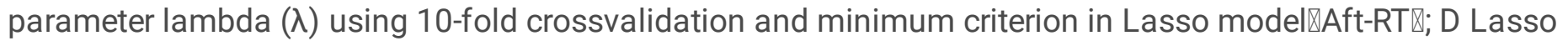
coefficient profiles of the 1037 radiomics features. The vertical gray line was drawn at the value selected using 10-fold cross-validation $₫$ Aft-RT叉 

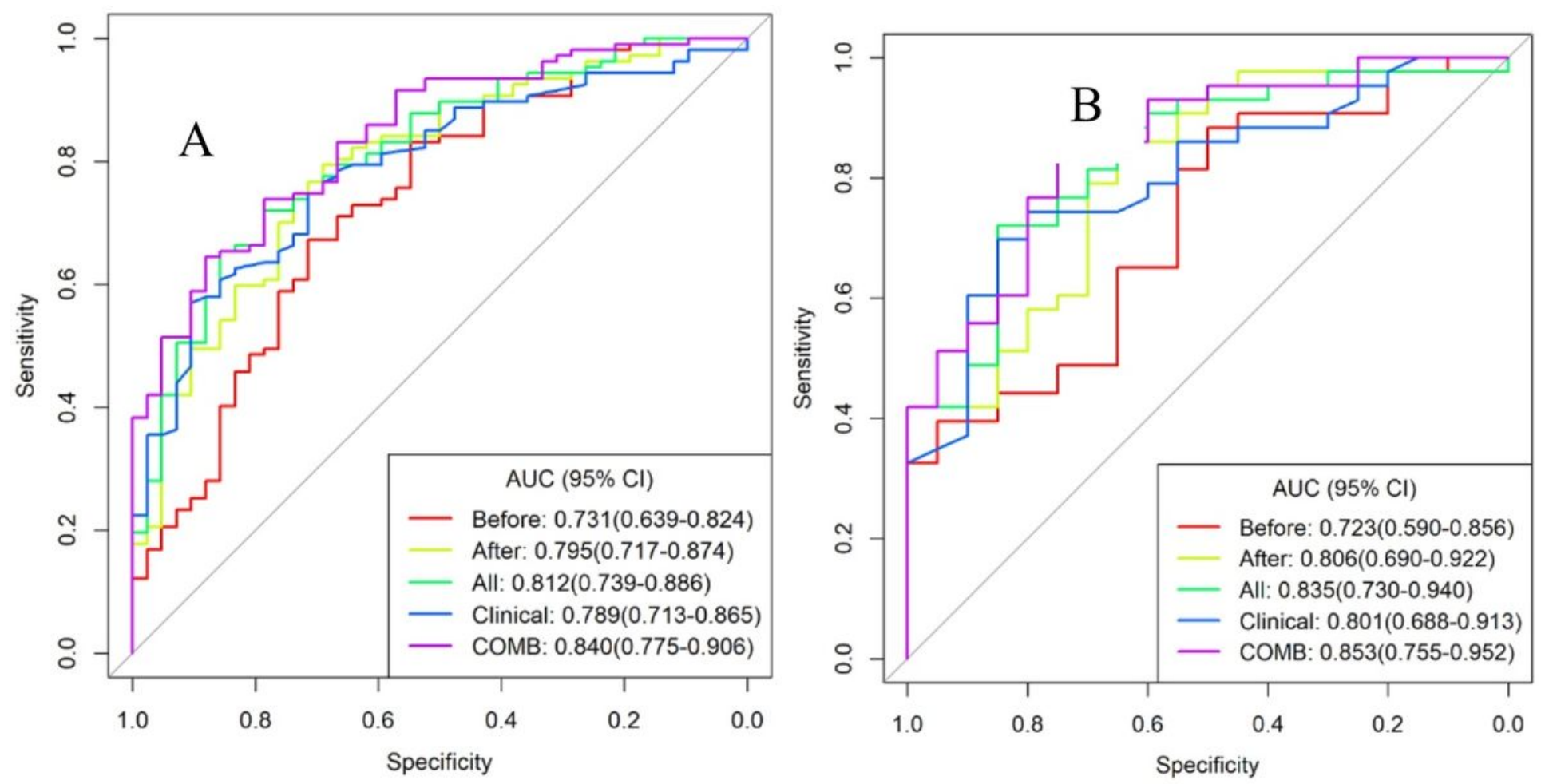

Figure 2

The efficacy of different models in predicting short-term outcomes after radiotherapy for esophageal cancer were evaluated using ROC. (A) training group; (B)validation group Before: radiological label囚Pre-RT囚; After: radiological label『Aft-RT囚; All: radiological label『combination囚; Clinical: Clinical character; COMB: radiomics nomogram 


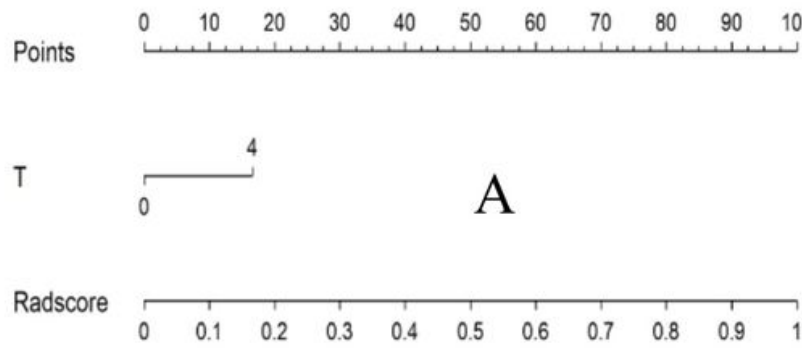

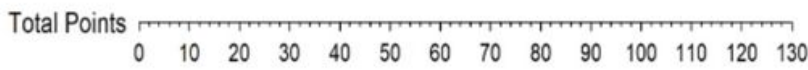

Risk
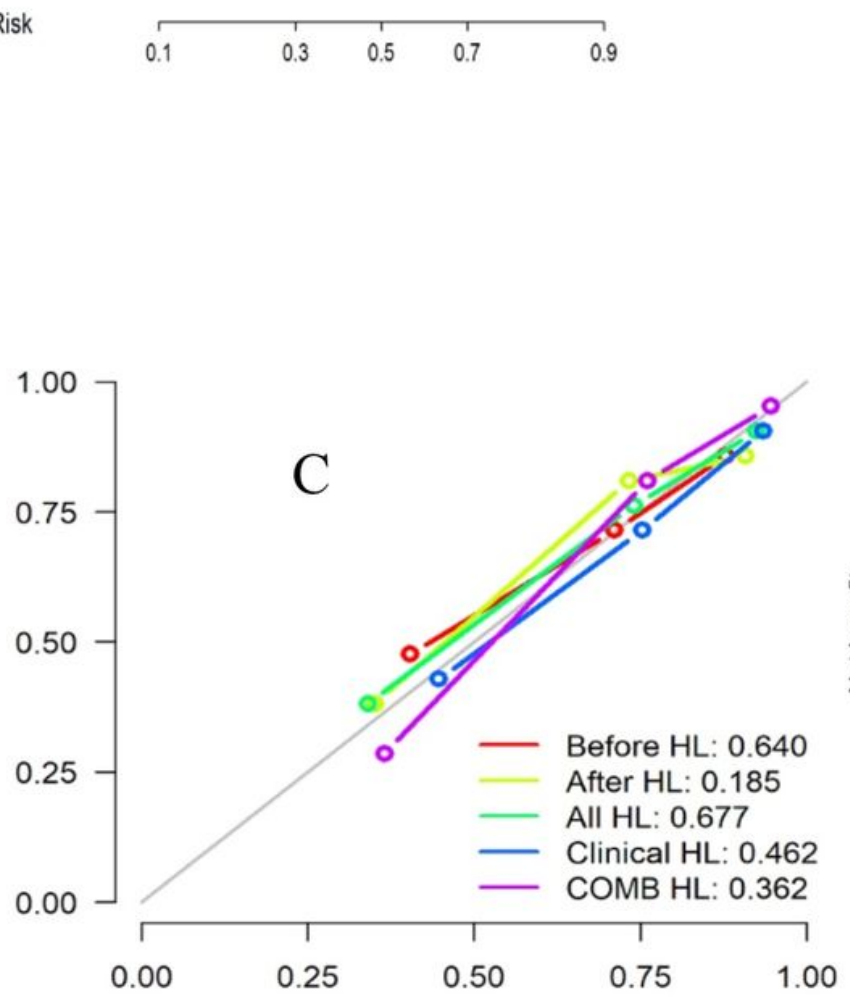

Predicted event probability

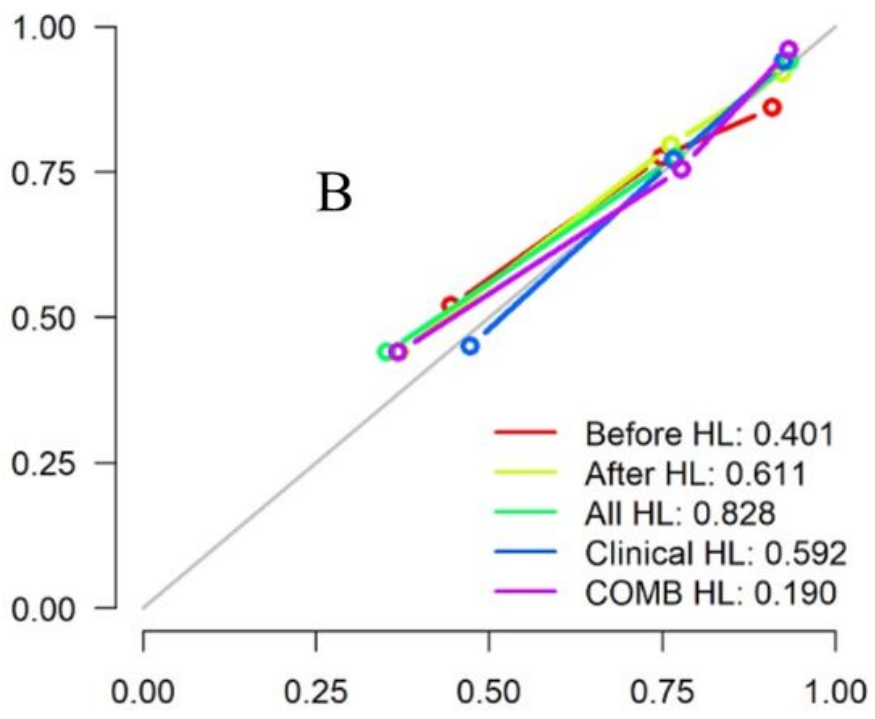

Predicted event probability

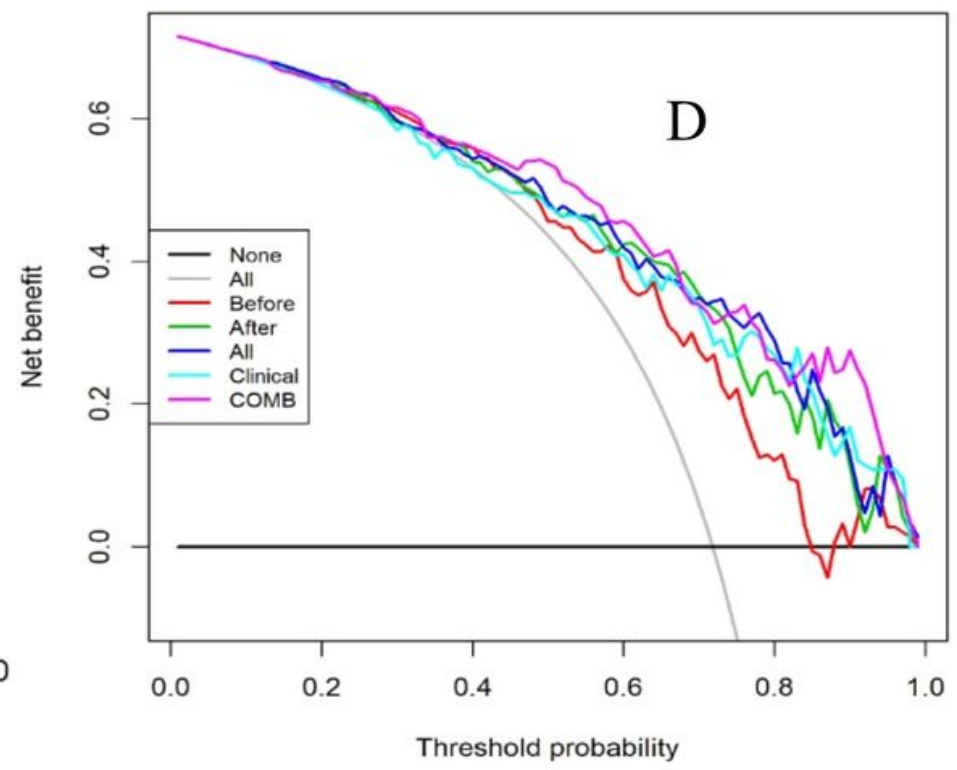

\section{Figure 3}

Radiomics nomogram for predicting CR of ESCC patients after radiotherapy. T: 0,T1-3 ; 2, T4 (A). Calibration curves of the radiomics nomogram in the training (B) and validation (C) cohorts. Potential incremental values of the radiomics nomogram relative to the radiological label were evaluated by net reclassification improvement (NRI) (D). 